\title{
Recent Advances in the Treatment of Arrhythmias
}

\author{
Augustus O Grant, MD
}

\begin{abstract}
Advances in endocardial mapping techniques and ablation have greatly increased the indications for catheterablation of supraventricular arrhythmias. Rate or rhythm control is a valid treatment option for patients with atrial fibrillation; however, all patients with one or more risk factors should be treated with oral anticoagulants. The early success rate and long-term cure of atrial fibrillation by radiofrequency catheter ablation continues to increase. The number of centers offering this treatment option has increased substantially. Implantable defibrillator-cardioverters are the primary treatment modality for patients with ventricular tachycardia and their role in primary prevention is also being defined. Future advances in arrhythmia management will include improvements in catheter design and energy sources for ablation, and greater monitoring capacity of implantable devices. (Circ J 2003; 67: 651-655)
\end{abstract}

Key Words: Atrial fibrillation; Catheter ablation; Implantable cardioverter-defibrillator

$\mathbf{T}$ he treatment of cardiac arrhythmias remains a major clinical challenge, but recent advances have expanded the scope of treatment options that patients can be offered. Because the strategies that are emphasized in the management of supraventricular and ventricular arrhythmias differ significantly, these 2 broad groups of arrhythmias will be discussed separately.

\section{Supraventricular Arrhythmias}

\section{Paroxysmal Supraventricular Tachycardia (PSVT)}

Radiofrequency catheter ablation (RF-CA) has been widely adopted as the treatment for PSVT resulting from accessory atrioventricular (AV) pathways, re-entry within the AV node and ectopic atrial tachycardia. Table 1 summarizes the published experience with catheter ablation of accessory AV connections. Most of the data are not new and the recurrence rate is almost certainly lower in 2003. What is significant about RF-CA for these arrhythmias is its greater availability. The relatively low procedural risk and the high likelihood of cure have provided the momentum for early and more widespread use of ablation. Radiofrequency catheter ablation may be considered the primary treatment for many supraventricular arrhythmias (eg, WPW syndrome). By its very nature, RF-CA requires a precise definition of the arrhythmia mechanism and the anatomic substrate. The latter is particularly difficult in complex cases.

Re-entry using accessory AV pathways or maintained within the AV node occurs in predictable anatomic locations in the vicinity of the AV groove. Single catheter mapping using multiplane fluoroscopy around the tricuspid ring and the coronary sinus is sufficient to give precise anatomic localization. This is usually not the case with ectopic atrial tachycardias. The early activation and rapid spread of activity over the normal atrium limits the precision of foci localization. For arrhythmias arising in right atriotomy

(Received May 30, 2003; accepted May 8, 2003)

Cardiology Division, Duke University, Durham, NC, USA

Mailing address: Augustus Grant, MD, Box 3504, Duke Medical

Center, Durham, NC 27710, USA. E-mail: grant007@mc.duke.edu scars, the site of re-entry is usually identified by the localization of areas of absent or double potentials. With single catheter mapping it may be difficult to reposition the catheter at previously defined sites for subsequent RF energy application.

Electro-anatomic mapping has greatly facilitated the approach to these arrhythmias. The CARTO system uses low-intensity magnetic fields to localize an endocardial mapping/ablation catheter in 3-dimensional (D) space5,10 Locator coils placed under the table emit the low-level magnetic fields. The endocardial mapping catheter has a location sensor proximal to the electrodes. The catheter has radiofrequency (RF) delivery capabilities and a thermocouple. Location of the sites of RF application can be stored. It can be used with most commercial RF generators. Dragging the catheter over the myocardium permits the simultaneous acquisition of the electrogram and location of the catheter tip relative to a reference catheter. From the acquired data, a 3-D color-coded electro-anatomic map can be constructed. Anatomical boundaries of the chamber being mapped are obtained under fluoroscopic guidance; for example in the right atrium, these sites could be the superior and inferior vena cavae, the os of the coronary sinus and the tricuspid annulus. Resolution is of the order of $1 \mathrm{~mm}$. A particular advantage of the system is that the 3-D map can be rotated in any direction. The reconstruction of the chamber may involve either activation or voltage. Focal origin of an arrhythmia is registered as radial spread of excitation; reentrant excitation is registered as contiguous lines of early and late excitation and the range of activation times that approximate the cycle length of the arrhythmia. Electroanatomic mapping has been particularly useful in localizing atrial arrhythmias to the scar of a prior atriotomy or left atrial anastomoses following lung transplantation.

\section{Atrial Fibrillation and Flutter}

The scope of the problem and the complexity of patient profiles make atrial fibrillation a continuing challenge; for example, $2.5 \%$ of individuals aged over 65 years are affected and the percentage rises to $10 \%$ in those aged 80 years or older6 As the population in the developed countries ages, the prevalence is expected to double in the next 
Table 1 Clinical Success of Radiofrequency Catheter Ablation of Accessory AV Connections in Patients With WPW Syndrome or Concealed Accessory Pathways

\begin{tabular}{lccccc}
\hline \hline Authors & Year & No. of patients & Acute success (\%) & Complications (\%) & Recurrence rate \\
\hline Jackmen et al & 1991 & 166 & 99 & 3 & 9 \\
Calkins et al & 1991 & 56 & 93 & 2 & 2 \\
Schbrser et al & 1991 & 92 & 86 & 3 & 3 \\
Calkins et al & 1999 & 500 & 93 & 3 & 8 \\
Kay et al & 1993 & 363 & 95 & 1 & 0
\end{tabular}

Adapted from Haines DE. Catheter ablation for arrhythmias. Philadelphia: Lippincott Williams \& Wilkies, 2002.

\section{Mortality in the Rhythm-Control Group and the Rate-Control Group}

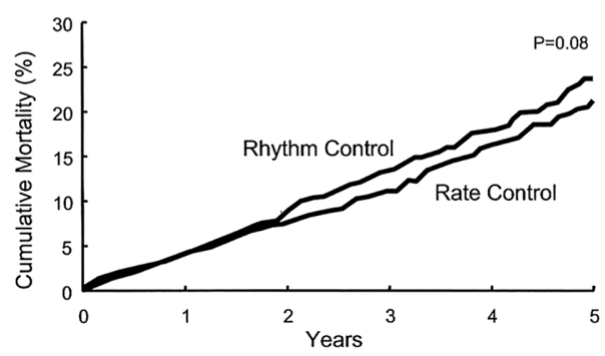

Fig 1. Mortality in the rhythm-control and rate-control groups in the AFFIRM trial (Adopted from N Engl J Med 2002; 347: 1825-1833).

20 years. The major therapeutic objectives in patient management are not controversial and include the following.

(a) Relief of symptoms through rate control, and the restoration and maintenance of normal sinus rhythm.

(b) Prevention of the thrombo-embolic complications.

(c) Prevention of tachycardia-induced cardiomyopathy.

Rate control has the advantage that it is relatively safe and non-pharmacologic strategies are highly effective. However, the rate is frequently not well controlled and substantial effort is required on the part of the patient and healthcare provider. Restoration and maintenance of normal sinus rhythm offers better symptom relief, provides a hemodynamic advantage in the presence of left ventricular inflow and outflow obstruction (eg, mitral stenosis and hypertrophic cardiomyopathy) and prevents tachycardiainduced cardiomyopathy. It may also reduce the stroke risk. The long-term efficacy of maintaining normal sinus rhythm is low. Drug initiation frequently requires hospitalization and long treatment carries a significant risk of adverse reaction to antiarrhythmic drugs4 The usual treatment strategy for atrial fibrillation is to attempt restoration and maintenance of normal sinus rhythm. Only after repeated failures does one resort to rate control and longterm anticoagulation.

Two major trials addressing the issue of rate versus rhythm control were recently published. The Atrial Fibrillation Follow-up Investigation of Rhythm Management (AFFIRM) trial was a large randomized trial conducted in the United States and Canada! The smaller Rate Control versus Electrical Cardioversion for persistent atrial fibrillation, or RACE trial, was a multicenter European trial ${ }^{21}$ The design of the trials was instructive of the contemporary approach to the management of atrial fibrillation and has major implications for treatment. For this reason, I shall recount details of the trial design and results?
AFFIRM randomized 4,006 patients of mean age 70 years to the rate control or rhythm control strategy. The pharmacologic approaches for rate control included $\beta$-adrenergic blockers, verapamil, diltiazem or digoxin alone or in combination. Target heart rate was addressed by 24-h Holter recording with a resting target heart rate of $\leq 80$ beats $/ \mathrm{min}$, on the 6 -min walk, $<110$ beats/min and no individual rate greater than $110 \%$ of the age-predicted heart rate. The non-pharmacologic rate control strategies included radiofrequency $\mathrm{AV}$ node modification or ablation and pacemaker implantation. For the rhythm control strategy, antiarrhythmic drug choice was at the discretion of the primary physician. However, there were some guidelines. Disopyramide was not used if the ejection fraction was less than 30\%. Class I antiarrhythmic drugs were avoided in patients with congestive heart failure, left ventricular hypertrophy or coronary artery disease. Sotalol was avoided in patients with asthma, congestive heart failure and patients with an ejection fraction of less than $25 \%$ or QT interval prolongation. Amiodarone was the most widely used antiarrhythmic drug. All patients received AV nodal blocking drugs. Non-pharmacologic strategies included catheter ablation for atrial flutter/fibrillation, the surgical maze procedure and single or multisite atrial pacing.

In the rate control strategy, warfarin therapy was continuous with a target INR of 2-3. In the rhythm control group, the level of anticoagulation was the same, but could be discontinued after 4-12 weeks of normal sinus rhythm. Repeated cardioversion required repeat anticoagulation. There was substantial crossover between the 2 trial groups, emphasizing that neither strategy is entirely satisfactory. The mean duration of follow-up was 3.5 years.

The essence of the trial results is summarized in Fig 1. There was no statistically significant difference in cumulative mortality between the strategies. In fact, the trend was for better survival in the rate control group. Strokes occurred at an overall rate of $1 \%$ per year. It was most likely to occur if anticoagulation was suboptional. The data suggest that continuous anticoagulation should be maintained in both patient groups.

The RACE trial randomized 522 patients with recurrent persistent atrial fibrillation or flutter. The composite endpoint was death from cardiovascular disease, thromboembolic complications, and bleeding or pacemaker implantation. The conclusions were similar to AFFIRM: rhythm control had no advantage over rate control. Five of 6 patients in the rhythm control group who had a stroke after discontinuation of warfarin were in normal sinus rhythm. Together, the 2 trials suggest that the rate control strategy should be used earlier as a treatment strategy. It need not be an approach of last resort. Also, anticoagulation should not be stopped even in patients with normal sinus rhythm.

The non-pharmacologic approaches to the management 
Contemporary Management of AF

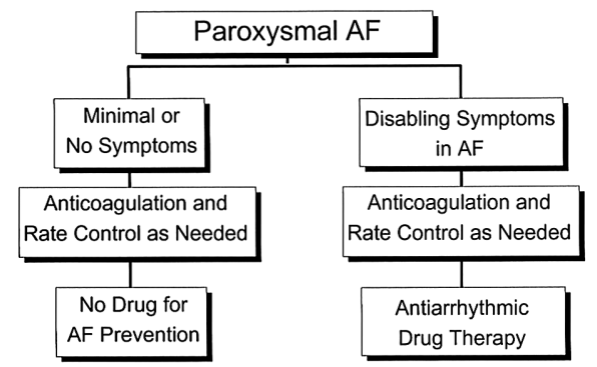

Fig 2. Algorithm for the contemporary management of atrial fibrillation.

of atrial fibrillation have been an area of very active investigation. The thrust of the effort has been the use of RF-CA for the cure of paroxysmal and chronic atrial fibrillation. The initial catheter based approach to the treatment of atrial fibrillation involved modification of the anatomic substrate. Linear lesions were performed in the right atrium to reduce the regions of myocardium to sizes below the critical mass required to maintain atrial fibrillation. The approach required long procedure times $(\geq 8 \mathrm{~h})$, had relatively low success rates and a high recurrence rate.

An alternative approach was to eliminate or modify the mechanisms that initiate atrial fibrillation. An accumulating body of evidence suggests most episodes of atrial fibrillation are initiated in venous structures, including the pulmonary veins, the superior vena cava, the vein of Marshall and the coronary sinus; $80-90 \%$ of foci are located in the pulmonary veins. The pulmonary veins are covered by myocardium sleeves that are $2-25 \mathrm{~mm}$ in length. These myocardial sleeves have an embryonic origin similar to conduction-system myocytes and are capable of impulse initiation by automaticity or triggered activity 19

The effort to cure atrial fibrillation by ablating the initiating foci was led by Haissaguerre et al8,9,12,13 The initiating focus was localized by activation mapping, which usually required the observation of frequent atrial premature beats or paroxysms of atrial fibrillation. Earliest local activity usually mapped to $2-4 \mathrm{~cm}$ within the main pulmonary veins or their branches. The frequency distribution of initiation foci was: left superior $>$ right superior $>$ left inferior $>$ right inferior pulmonary vein. The majority of patients had a focus in a single vein; some patients $(\sim 30 \%)$ had foci in one or more veins. After a mean of almost $1.5 \mathrm{RF}$ procedures, $70 \%$ of patients were cured. This initial approach of ablation within the pulmonary veins had several drawbacks:

(a) the presence of multiple foci in some patients,

(b) emergence of new foci after the initial procedure,

(c) lack of spontaneous or inducible atrial fibrillation or atrial tachycardia at the time of electrophysiology study,

(d) mapping may require repeated cardioversions of episodes of sustained atrial fibrillation. Internal cardioversion may be a useful option during the procedure.

Many of these problems could be obviated by circumferential ablation of the vein ostia. The inclusion of all 4 ostia would remove the need for localization of earliest activation to a specific vein. This approach was supported by the initial reports of vein isolation. A major complication of the procedure is pulmonary vein stenosis, which made isolation of veins that did not show early activation unde-

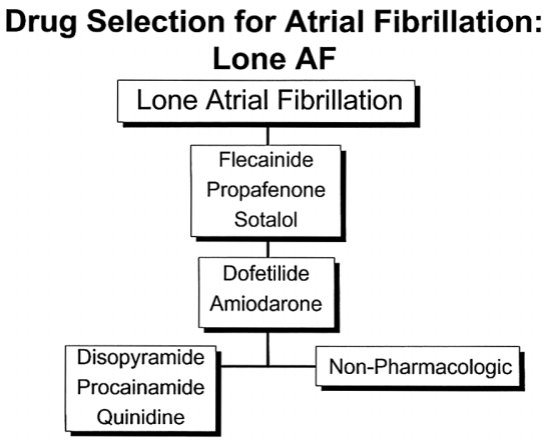

Fig 3. Algorithm for drug selection for patient with atrial fibrillation (AF) and no heart disease.

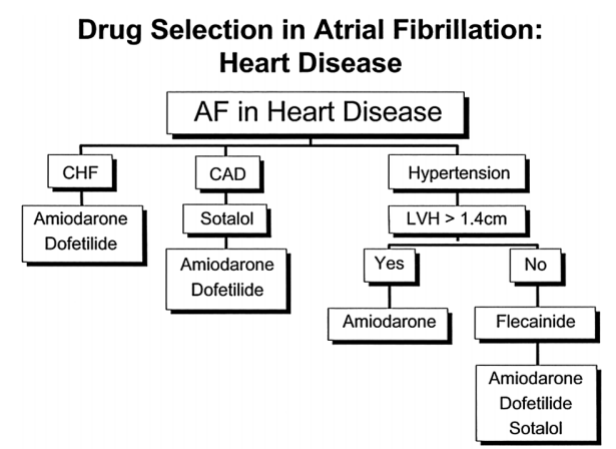

Fig 4. Algorithm for the management of patients with atrial fibrillation and structural heart disease. CHF, congestive heart failure; CAD, coronary artery disease; LVH, left ventricular hypertrophy.

sirable.

Further observations suggest that complete isolation of each vein may not be necessary. The myocardial sleeve may not be present over the entire circumference of the vein. Ablation could be targeted to those vein segments where myocardial fibers were present. In the majority of cases, ablation of less than $50 \%$ of the vein circumference effected isolation. When accompanied by a reduction of the $\mathrm{RF}$ energy to $20-30 \mathrm{~W}$, the incidence of pulmonary vein stenosis could be reduced to approximately $1 \%$. Symptomatic pulmonary vein stenosis requires dilation and stenting. The other complications are thrombo-embolism and pericardial effusions. Success rates of $80-90 \%$ have been reported for both paroxysmal and chronic atrial fibrillation. With such high success rates, the current trend is to relax the stringency of the indications for catheter ablation of atrial fibrillation.

Surgical ablation aimed at reducing the mass required to support atrial fibrillation preceded catheter-based strategies. The potential morbidity of the procedure and the paucity of centers with significant experience have limited the application of the procedure. The use of minimally invasive techniques may reduce the morbidity of the procedure. At the present time, the principal indication for the surgery is its combination with a planned procedure, such as mitral valve repair or replacement, or coronary artery bypass grafting 22

Local conduction delays may contribute to the recurrence or persistence of atrial fibrillation. Dual-site pacing may result in more homogeneous conduction. A number of studies have shown that dual-site pacing (eg, from the high right atrium and the coronary sinus) can reduce the fre- 
quency and duration of episodes of atrial fibrillation. ${ }^{15,18}$ It may be appropriate to use dual-site atrial pacing in patients with other indications for pacemaker implantation. The available data do not support implantation of the pacemaker solely for this perceived benefit.

The results of the AFFIRM and RACE trials, and the non-pharmacologic strategies, provide the basis for development of treatment algorithms (Figs 2-4) for the management of atrial fibrillation. Important determinants of the approach include the level of patient symptoms, the presence and type of structural heart disease, and the local expertise.

\section{Sudden Death and Ventricular Tachycardia/Fibrillation}

The challenge that sudden death presents is amply illustrated by the work of Huikuri et al!1 They summarize the relationship between the various risk groups and the number of individuals in each of these groups who will suffer sudden death. The incidence of sudden death is highest in patients with a prior myocardial infarction, low ejection fraction and a history of ventricular tachycardia. Clearly, all these characteristics are well-established risk factors for sudden death. However, they account for the minority of total sudden deaths in the population. In contrast, the general population as a whole has a very low incidence of sudden death. However, because of the large number of individuals that are at risk, the majority of sudden deaths occur in this population. There are few tests with the potential for screening the general population for sudden death risks and there are no therapies suitable for application to large numbers of low-risk individuals. A more fruitful approach focuses on the intermediate group in which a number of established risk factors can be identified and for which there are therapies that prevent a substantial number of sudden deaths. The MADIT II trial presented by Moss et al at the American College of Cardiology meeting in 2002 is the first study to document effective primary prevention against sudden death ${ }^{16}$ To be included in the study, patients had to be older than 21 years of age with a documented history of myocardial infarction and a left ventricular infraction ejection fraction $\leq 30 \%$ within the preceding 3 months. Note that antecedent cardiac arrhythmias, such as nonsustained ventricular tachycardias, were not required for inclusion into the study. Exclusion criteria included indications for implantable cardioverter-defibrillator implantation, New York Heart Association functional class IV, acute myocardial infarction during the preceding month and advanced cerebrovascular disease. The study was a sequential design. Boundary conditions indicating the greater efficacy of implantable cardioverter-defibrillator were crossed in November 2001, and the study was closed out in January 2002. After an average follow-up of 20 months, there was a reduction in the death rate of approximately $25 \%$ in the implantable cardioverter-defibrillator group.

In the United States, the result of this study will have an enormous impact on healthcare costs. At the time that the study was reported, it was estimated that nationwide, 300,000 patients would meet the implantation criteria, but perhaps only one-third would receive the device. At an average cost of $\$ 30,000$ per device implantation, the total added cost for the healthcare bill would be $\$ 3$ billion. In an incisive editorial published in Circulation, Zipes raised the question as to whether a 'Volkswagen or Rolls Royce' is required for a preventive indication ${ }^{23}$ Simply put, for primary prevention, is it not more reasonable to use a simple shock box capable of delivering 8-10 discharges without the added circuitry for pacing and electrogram storage? The circuitry of the implantable cardioverter-defibrillator is probably not the major component of cost. The sales staff and extensive support services that the device companies provide adds substantially to overall costs.

Following the publication of the AVID and MADIT I trials, the implantable cardioverter-defibrillator has supplanted antiarrhythmic drugs as the preferred treatment for ventricular tachycardia. The major manufacturers have greatly widened the scope of diagnostics and therapies that the devices can provide! ${ }^{7}$ Storage of relevant electrograms is now standard. Approximately $80 \%$ of monomorphic ventricular tachycardias are pace terminable, whereas most polymorphic ventricular tachycardias require cardioversion. Devices that distinguish the 2 forms of ventricular tachycardia can provide tiered therapy with longer battery life.

Many patients with ventricular tachycardia (VT) or fibrillation (VF) have a significant burden of concomitant atrial fibrillation. Dual-chamber atria-ventricular implantable cardioverter-defibrillators are now available for treating VT/VF patients with drug resistant atrial fibrillation. Algorithms enable the device to distinguish atrial and ventricular fibrillation and deliver appropriate therapy. The clinical benefit of biventricular pacing in patients with advanced heart failure and marked QRS prolongation is established. The biventricular pacing/implantable cardioverter-defibrillator system is a new therapeutic option for patients who meet the criteria for implantable cardioverterdefibrillator and biventricular pacing. Radiofrequency catheter ablation remains an important therapeutic option in subgroups of patient where it is curative; for example, patients with right ventricular outflow tract tachycardia and bundle branch re-entrant ventricular tachycardia. Patients with ventricular tachycardia related to structural heart disease are usually treated with implantable cardioverterdefibrillators. However, frequent implantable cardioverterdefibrillator discharges may compel other therapeutic measures such as RF-CA, which in patients with structural heart disease presents a number of special challenges:

(a) The large size of the re-entrant circuits.

(b) An average of 3 ventricular tachycardia morphologies in each patient.

(c) Hemodynamic instability during VT, limiting conventional single-catheter mapping.

(d) Mid-myocardial and subepicardial location of some arrhythmias.

(e) The presence of endocardial clot.

A number of strategies have been introduced over the past several years to address some of these issues. Cooledtip RF catheters have been introduced to produce larger and deeper ablations. In the closed system, cooled saline is recirculated through the electrode tip at $0.6 \mathrm{ml} / \mathrm{s}$; in the open system the cool saline passes directly into the circulation. RF application can be performed to electrode temperatures of $40-50^{\circ} \mathrm{C}$. Using this system, Calkins et al reported freedom from VT for a mean of 1 year in $50 \%$ of 146 patients undergoing ablation for ventricular tachycardia using the saline-cooled catheter; however, the complication rate was high and included 4 deaths?

In approximately $15 \%$ of patients, the re-entrant circuit for ventricular tachycardia is epicardial. Potential access to 
the epicardium includes direct vision at cardiac surgery, through catheterization of the cardiac veins or through pericardial puncture. Direct vision at surgery implies a concomitant thoracotomy. Only limited regions of the myocardial surface are accessible by venous catheterization. Sosa et al reported their recent experience with direct pericardial puncture ${ }^{20}$ An epidural needle is used to enter the pericardium by a subxiphoid puncture and an electrode catheter can then be positioned in the pericardial space, verifying its position by the injection of 1-2 ml of contrast, and by reference catheters in the coronary sinus. High-quality stable electrograms can be obtained using this approach. The ventricular tachycardia circuit is defined with the aid of brief RF pulses that terminate the tachycardia transiently. A prolonged application of RF energy is then applied to effect permanent ablation of the ventricular tachycardia. Sosa et al report a success role of $60 \%$. The complications include cardiac puncture in approximately $10 \%$ of patients, and the potential for damage to the coronary arteries at sites in close proximity $(<12 \mathrm{~mm})$. Prior pericardiotomy for cardiac surgery precludes use of the technique.

Marchlinski et al have recently described a strategy for the RF ablation of ventricular tachycardia associated with hemodynamic instability 14 Areas of abnormal endocardium are recognized by low voltages recorded with an electroanatomic (CARTO) mapping system. Linear ablation lesions extended from regions of relatively dense scar (low level signals on the voltage maps) to anatomic boundaries or to normal myocardium. The linear lesion was also positioned at candidate sites when pacing reproduced the ventricular tachycardia morphology on the 12-lead ECG (ie, by pace mapping). During a median follow-up of 8 months, 12 of 14 patients were free of arrhythmia. Radiofrequency catheter ablation retains a place in the management of ventricular tachycardia despite implantable cardioverter-defibrillator and drug therapy.

\section{References}

1. AFFIRM. A comparison of rate control and rhythm control in patients with atrial fibrillation. $N$ Engl J Med 2002; 347: 1825-1833.

2. AFFIRM. Atrial Fibrillation Follow-Up Investigation of Rhythm Management: The AFFIRM Study Design. Am J Cardiol 1997; 79: $1198-1202$

3. Calkins H, Epstein A, Packer D, Arria AM, Hummel J, Gilligan DM, et al. Catheter ablation of ventricular tachycardia in patients with structural heart disease using cooled radiofrequency energy. $J \mathrm{Am}$ Coll Cardiol 2000; 35: 1905-1914.

4. Coplen SE, Antman EM, Berlin JA, Hewitt P, Chalmers TC. Efficacy and safety of quinidine therapy for maintenance of sinus rhythm. Circulation 1990; 82: 1106-1116.

5. Darbar D, Olgin J, Miller J, Friedman PA. Localization of the origin of arrhythmias for ablation: From electrocardiography to advanced endocardial mapping systems. J Cardiovasc Electrophysiol 2001; 12: $1309-1325$.

6. Go AS, Hylek EM, Phillips KA, Chang Y, Henault LE, Selby JV, Singer DE. Prevalence of diagnosed atrial fibrillation in adults: National implications for rhythm management and stroke prevention: The AnTicoagulation and Risk Factors in Atrial Fibrillation (ATRIA) Study. JAMA 2001; 285: 2370-2375.

7. Haines DE. Catheter ablation therapy for arrhythmias. Philadelphia, PA: Lippincott Williams \& Wilkins, 2002.

8. Haissaguerre M, Jais P, Shah DC, Takahashi A, Hocini M, Quiniou $\mathrm{G}$, et al. Spontaneous initiation of atrial fibrillation by ectopic beats originating in the pulmonary veins. N Engl J Med 2000; 339: 659666.

9. Haissaguerre M, Jais P, Shah DC, Takahashi A, Hocini M, Quiniou $\mathrm{G}$, et al. Electrophysiological end point for catheter ablation of atrial fibrillation iniitated from multiple pulmonary venous foci. Circulation 2000; 101: 1409-1417.

10. Hoffmann E, Nimmermann P, Reithmann C, Elser F, Remp T, Steinbeck G. New mapping technology for atrial tachycardias. $J$ Intervent Card Electrophysiol 2000; 4: 117-120.

11. Huikuri HV, Castellanos A, Myerburg RJ. Sudden death due to cardiac arrhythmias. N Engl J Med 2001; 345: 1473-1482.

12. Jais P, Shah DC, Haissaguerre M, Hocini M, Garrigue S, Clementy J. Atrial fibrillation: Role of arrhythmogenic foci. J Interv Card Electrophysiol 2000; 4: 29-37.

13. Jais P, Weerasooriya R, Shah DC, Hocini M, Macle L, Choi K-J, et al. Ablation therapy for atrial fibrillation (AF): Past, present and future. Cardiovasc Res 2002; 54: 337-346.

14. Marchlinski FE, Callans DJ, Gottlieb CD, Zado E. Linear ablation lesions for control of unmappable ventricular tachycardia in patients with ischemic and nonischemic cardiomyopathy. Circulation 2000; 101: $1288-1296$.

15. Mirza I, James S, Holt P. Biatrial pacing for paroxysmal atrial fibrillation: Randomized prospective study into the suppression of paroxysmal atrial fibrillation using biatrial pacing. J Am Coll Cardiol 2002; 40: 457-463.

16. Moss AJ, Zareba W, Hall J, Klein H, Wilber DJ, Cannom DS et al. Prophylactic implantation of a defibrillator in patients with myocardial infarction and reduced ejection fraction. $N$ Engl J Med 2002; 346: $877-883$.

17. Saksena S, Madan N. Management of the patient with an implantable cardioverter-defibrillator in the third millennium. Circulation 2002; 106: $2642-2646$

18. Saksena S, Prakash A, Ziegler P, Hummel JD, Friedman P, Plumb VJ, et al. Improved suppression of recurrent atrial fibrillation with dual-site right atrial pacing and antiarrhythmic drug therapy. $J \mathrm{Am}$ Coll Cardiol 2002; 40: 1140-1150.

19. Scheinman MM, Morady F. Nonpharmacological approaches to atrial fibrillation. Circulation 2001; 103: 2120-2125.

20. Sosa E, Scanavacca M, d'Avila A. Transthoracic epicardial catheter ablation to treat recurrent ventricular tachycardia. Curr Cardiol Rep 2001; 3: 451-458.

21. Van Gelder IC, Hagens VE, Bosker HA, Kingma JH, Kamp O, Kingma T, et al. A comparision of rate control and rhythm control in patients with recurrent persistent atrial fibrillation. $N$ Engl J Med 2002; 347: 1834-1840.

22. Williams MR, Stewart JR, Bolling SF, Freeman S, Anderson JT, Argenziano M, et al. Surgical treatment of atrial fibrillation using radiofrequency energy. Ann Thorac Surg 2001; 71: 1939-1944.

23. Zipes DP. Implantable cardioverter-defibrillator: A Volkswagen or a Rolls Royce: How much will we pay to save a life? Circulation 2001; 103: $1372-1374$. 